\title{
The Role of Prophylactic Antibiotics for Percutaneous Procedures in Orthopaedic Surgery
}

\author{
A. Gulati ${ }^{*}$, A. Dixit ${ }^{2}$, D. M. Williamson ${ }^{1}$ \\ ${ }^{1}$ Great Western Hospital, Swindon, UK \\ ${ }^{2}$ University Hospital of Leicester, Leicester, UK \\ E-mail: 'gulatiaashish@doctors.org.uk \\ Received May 5, 2011; revised June 30, 2011; accepted July 8, 2011
}

\begin{abstract}
Introduction: This study investigates the current practice of surgeons in the United Kingdom with regards to their usage of prophylactic antibiotics for percutaneous orthopaedic procedures. Methods: An electronic survey of 10 questions was devised and sent to all members of the British Orthopaedic Association. Three hundred and three replies were obtained (172 consultants, 131 trainees). Results: Only half the numbers of orthopaedic surgeons would routinely use antibiotics for percutaneous K-wire fixation. Of the other half, $28 \%$ would never prescribe antibiotics and 22\% would use them in special circumstances only. These 'special circumstances' were also not standardised. $92 \%$ of those who did prescribe antibiotics would administer single dose only and the majority (90\%) would administer them during induction. There was no significant difference between trainees and consultants or between different orthopaedic procedures with regards to whether prophylactic antibiotics were prescribed or not. Discussion: This survey highlights the split of opinion amongst practising orthopaedic surgeons as to the necessity or otherwise of antibiotic prophylaxis in percutaneous orthopaedic procedures. There are no reliable guidelines and further work should be carried out to investigate this subject.
\end{abstract}

Keywords: Prophylactic Antibiotics, Percutaneous Procedures, K-Wires

\section{Introduction}

Infections in orthopaedics and trauma surgery are particularly challenging due to changing epidemiology and bacteriology of microbes, and the propensity for longterm morbidity. The issue is further complicated by rapidly increasing prevalence of antimicrobial resistance.

Surgical site infections are a major source of postoperative illness, accounting for nearly $25 \%$ of all nosocomial infections in the United States each year. The estimates from the Centre for Disease Control and Prevention (CDC) suggest that approximately 500,000 surgical site infections occur annually in the United States [1]. In the United Kingdom, an estimated 320,000 patients acquire one or more hospital acquired infections every year during their in-patient stay, and these infections cost the hospital sector an estimated £930 million per year [2].

Numerous exogenous and endogenous factors have been implicated in contributing to the onset of bone and joint infection thereby resulting in increased morbidity, hospital stay, and health care costs. Hence, it is not uncommon in many situations in orthopaedic surgery to prescribe prophylactic antibiotics, particularly when carrying out an invasive procedure. However, inappropriate use of antibiotics may not actually prevent postoperative infections but instead it may contribute to the development of antibiotic resistance. This may then predispose patients to further infections, increasing the risk of adverse reactions and healthcare costs [3].

The use of prophylactic antibiotics is considered routine in some orthopaedic procedures such as joint replacement [4], spine surgery and major fracture fixation. There are, however, no clear guidelines [5,6] about using prophylactic antibiotics for percutaneous Kirschner wires (K-wires) in orthopaedic surgery. This aim of this study was to investigate the current practice of orthopaedic surgeons in the United Kingdom (UK) with regards to the use of prophylactic antibiotics when carrying out procedures which involve the insertion of percutaneous K-wires. 


\section{Methods}

An electronic survey was devised and sent to all members of the British Orthopaedic Association. A further reminder, two weeks after the first invitation, was sent so as to increase the response rate. Three hundred and four replies were obtained of which 172 were from consultants and 132 were from surgeons of other grades (nontraining career-grade orthopaedic surgeons and trainees).

The statistical analyses of responses was carried out using Chi squared, Pearson's correlation co-efficient and Spearman correlation, where indicated, to test for significance. The $\alpha$-level of significance was defined as 5\%. SPSS $^{\circledR}$ 12.0.1 for Windows (SPSS Inc., Chicago, IL, USA) software was employed for statistical analysis of the data.

\section{Results}

The first part of the analysis was to assess whether the obtained responses allow a homogenous comparison of the representative data.

Fifty-seven percent consultants, 39\% trainee registrars, $3 \%$ non-training career-grade surgeons and $1 \%$ Senior House Officers (SHOs) participated in the survey (Table 1). In terms of clinical practice, $20 \%$ had 0 - 5 years, $31 \%$ had 5 - 10 years, $22 \%$ had $10-15$ years, $12 \%$ had 15 - 20 years and $15 \%$ had $>20$ years of orthopaedic experience. The responses were obtained from trauma and orthopaedics surgeons covering almost all the subspecialties (16\% hip, $13 \%$ upper limb, $11 \%$ knee, $8 \%$ hands, $8 \%$ foot and ankle, $6 \%$ trauma, $6 \%$ general orthopaedics, $1 \%$ spine, $1 \%$ orthopaedic oncology and $24 \%$ trainees with no primary subspecialty interest) at the time of participation. The responses, therefore, came from a sample representative of differing grades of orthopaedic surgeons, surgical experience and subspecialty interest setting a platform for meaningful statistical analysis.

The results of the survey demonstrate a mixture of opinion and non-uniformity of current practice among the practicing orthopaedic surgeons. Overall 50\% would always use prophylactic antibiotics when carrying out percutaneous K-wires, $27 \%$ would never use them and $23 \%$ would use them only in special circumstances only (Table 2). These "special circumstances" were also not standardised and included open fractures, associated contaminated wounds, other co-morbidities, diabetes, paediatric growth plate fractures and fixation of foot fractures. One response read, "when I remember".

A subset analysis of those who did prescribe prophylactic antibiotics while using percutaneous K-wires demonstrated that $92 \%$ surgeons would prescribe only one dose while $2 \%$ would prescribe two doses and $6 \%$ would
Table 1. The responses from different grades of orthopaedic surgeon.

\begin{tabular}{ccc}
\hline Level & Frequency & Percent \\
\hline Consultant & 172 & 56.6 \\
Staff Grade/Associate Specialist & 9 & 3.0 \\
Registrar (trainees) & 119 & 39.1 \\
SHO & 4 & 1.3 \\
Total & 304 & 100 \\
\hline
\end{tabular}

Table 2. The number of participants using antibiotics.

\begin{tabular}{ccc}
\hline Antibiotics prescription & Frequency & Percent \\
\hline Never & 83 & 27.3 \\
Sometimes & 69 & 22.7 \\
Always & 152 & 50.0 \\
Total & 304 & 100 \\
\hline
\end{tabular}

prescribe three doses (Table 3). 98\% surgeons would prescribe antibiotics parenterally (IV or IM) at the time of induction of general anaesthesia (GA). Two percent surgeons would prescribe oral antibiotics at the time of discharge in addition to the ones prescribed at the time of induction.

The survey also investigated procedure-specific antibiotic prophylactic use. Eighty-five percent surgeons would prescribe prophylactic antibiotics for finger phalanx/metacarpal fracture stabilisation, $84 \%$ for metatarsal osteotomy and $82 \%$ when performing distal radius fracture fixation or toe fusion.

The second part of the analysis was to collate the results and assess whether the grade of the surgeon, their experience or their primary sub-specialist interest influenced the decision as to whether to use antibiotics or not and the manner in which they were prescribed.

No significant difference was demonstrated between trainees and consultants $(p=0.45)$ in terms of decision whether to prescribe antibiotics or not. The experience of the surgeon and sub specialist interest also did not influence this choice ( $p=0.34$ and $p=0.51$ respectively). There was, however, a significant difference $(p=0.04)$ in the number of doses prescribed with more consultants (Table 4) prescribing three doses of prophylactic antibiotics compared with trainees $(7.9 \%$ and $3.4 \%$ respectively). The more experienced surgeons ( $>20$ years experience) were five times more likely (Table 5) to prescribe three prophylactic doses $(p=0.05)$ when carrying out percutaneous K-wires.

The preferred route of administration was intra-venous (IV) at the time of general anaesthesia (GA) induction 
Table 3. The number of doses of prophylactic antibiotics being used.

\begin{tabular}{ccc}
\hline Number of doses & Frequency & Percent \\
\hline 1 & 204 & 92.2 \\
2 & 3 & 1.6 \\
3 & 14 & 6.2 \\
\hline
\end{tabular}

Table 4. The number of doses of prophylactic antibiotics prescribed by different grades of surgeons.

\begin{tabular}{ccccc}
\hline \multirow{2}{*}{ Level } & \multicolumn{3}{c}{ Number of doses } & Total \\
\cline { 2 - 4 } & $\mathbf{1}$ & $\mathbf{2}$ & $\mathbf{3}$ & \\
\hline $\begin{array}{c}\text { Consultant } \\
\text { Staff Grade/Associate } \\
\text { Specialist }\end{array}$ & 113 & 1 & 10 & 126 \\
Registrar & 64 & 1 & 0 & 6 \\
SHO & 1 & 0 & 1 & 2 \\
Total & 204 & 3 & 14 & 221 \\
\hline
\end{tabular}

Table 5. The number of doses of antibiotics prescribed as per the experience of the surgeon.

\begin{tabular}{ccccc}
\hline $\begin{array}{c}\text { Years practicing } \\
\text { Orthopaedics }\end{array}$ & \multicolumn{3}{c}{ Number of doses } & Total \\
\cline { 2 - 4 } & $\mathbf{1}$ & $\mathbf{2}$ & $\mathbf{3}$ & \\
\hline 0 to $5 \mathrm{yr}$ & 46 & 1 & 2 & 49 \\
5 to $10 \mathrm{yr}$ & 64 & 1 & 4 & 69 \\
10 to $15 \mathrm{yr}$ & 47 & 0 & 1 & 48 \\
15 to $20 \mathrm{yr}$ & 26 & 1 & 1 & 28 \\
$>20 \mathrm{yr}$ & 21 & 0 & 6 & 27 \\
Total & 204 & 3 & 14 & 221 \\
\hline
\end{tabular}

and was not dependent on the grade of the surgeon ( $p=$ $0.16)$, their experience $(p=0.39)$ or primary subspecialty $(p=0.61)$. In terms of the procedure-specific results, there was no significant difference between the grade of the surgeon, their surgical experience or their sub-specialist interest.

\section{Discussion}

Percutaneous K-wires are frequently used in a variety of clinical situations in orthopaedic surgery for temporary or permanent fixation of fractures and osteotomies [7]. When the wire is intended to stay in-situ for some time, there is a potential for micro-organism colonisation and infection. Few studies have recorded the incidence of infection following such intervention with ranges from zero to very low $[8,9]$ to rates of $6.6 \%[10], 6.8 \%$ [11] and $7.9 \%$ [12]. In cases where infection did occur, metalwork removal tended to lead to resolution $[13,14]$. It is, therefore, a common practice in many situations in medicine to prescribe prophylactic antibiotics whenever a foreign body is implanted into the body. We could not find any clear evidence to support this practice for percutaneous procedures in orthopaedic surgery.

It has been our experience that it depended largely on the surgeon's individual training/experience and preference as to whether or not to provide antibiotic prophylaxis for procedures requiring percutaneous K-wires to be left $i n$-situ for an extended period. We were surprised to find that no clear guidelines exist regarding this practice and were further surprised to find that literature pertaining to this issue is scant. The Scottish Intercollegiate Guidelines Network (SIGN) recommend [6] antibiotic prophylaxis should be administered in orthopaedic surgery if the procedure involves an insertion of a prosthetic device or any procedure where there is no direct evidence. The guidelines also recommend prophylactic antibiotics while carrying out an open reduction of a closed fracture. However, these guidelines do not specify whether this recommendation is applicable for percutaneously inserted K-wires for closed fractures. The National Institute for Health and Clinical Excellence (NICE) guidelines [5] suggest that antibiotic prophylaxis should be prescribed to patients before clean orthopaedic surgery if it involves a placement of a prosthesis or an implant. Again, these guidelines do not clearly provide advice about their usage for percutaneously inserted Kwires which are kept in-situ for few weeks only.

Our survey of practicing British orthopaedic surgeons reveals that $50 \%$ percent surgeons would always use prophylactic antibiotics while carrying out procedures involving percutaneous $\mathrm{K}$-wires. In contrast, $27 \%$ would never use them and $23 \%$ would use them only in special circumstances. These circumstances varied widely from surgeon to surgeon. This non-uniformity of practice is a clear reflection of lack of reliable and robust evidencebased guidelines on this topic. The number of doses of prophylactic antibiotics prescribed was further subject to inconsistency with the more experienced surgeons five times more likely to prescribe three prophylactic doses. In terms of the grade of the surgeon, $8.8 \%$ consultants prescribed three doses compared to $4.8 \%$ trainees $(p=$ 0.04 ). Although this was statistically significant, the number of surgeons prescribing three doses is probably too small to emphasise on this and most surgeons would use a single dose only.

The justification to use antibiotics prophylactically is perhaps based on the understanding that in surgical procedures that involve incorporation of an implant, the tissue-implant interface is especially prone to contamination. 
This combined with the disturbed bony structure and reduced local vascularity in trauma patients may impair the ability to obtain the appropriate antibiotic concentration locally [15]. Some in-vitro and in-vivo studies have shown that the local application of gentamicin, delivered from coating on titanium K-wires, can be effective in providing adequate prophylaxis [15]. Hargreaves et al. [16] and Rafique et al. [14] described a technique of burying the wires beneath the skin. This reduced the infective complication rate in these studies of isolated distal radius and hand fractures respectively. Lethaby et al. [17] performed a database review to investigate methods of pin-site care that might reduce infection rates but failed to find evidence of any particular strategy that might be superior to others with regard to infection rates. One of these trials [18] reported that infection rates were lower (9\%) with a regimen that included cleansing with half strength hydrogen peroxide and application of Xeroform dressing when compared with other regimens with different cleansing and dressing regimens (rates > 26\%). There was no evidence of a difference between groups in any of the other trials.

We are aware that this study has some limitations. The results represent the practice of just over 300 orthopaedic surgeons in the UK which is a relatively small percentage of the total. Furthermore, this survey only reflects the opinion of the members of the British Orthopaedic Association. Never the less, we believe that the participants in the survey provided a representative cohort for meaningful statistical analyses. There was a good mix of responses from all grades of orthopaedic surgeons encompassing all primary orthopaedic subspecialties. The main aim of this study was to highlight the fact that there are no evidence-based guidelines on this topic and that there is a split of opinion amongst the practicing orthopaedic community. Another weakness of the study is that the final analysis is based on the assumption that the trainees participating in the survey reflected their own practice and not of their hospitals or their orthopaedic teams.

\section{Conclusions}

This survey serves to highlight the split of opinion amongst practising orthopaedic surgeons as to the necessity or otherwise of antibiotic prophylaxis but we have also identified the lack of information available for clinicians on which to base this decision. We recommend that further work should be carried out to investigate this subject.

\section{Competing Interests}

The authors declare that they have no competing interests.

\section{Authors' Contributions}

AG and DMW helped in conducting the survey. AG and $\mathrm{AD}$ helped in writing up the paper, review of literature and statistical analysis. DMW supervised the whole project and the survey. DMW also edited and modified the final manuscript. All authors have read and approved the final manuscript.

\section{Acknowledgements}

The authors wish to acknowledge all the BOA members who participated in the survey. A special thanks to Mr J. Arbuthnot who helped in collecting the data and writing up the paper. No funding or grant has been received for the subject or content of the study.

\section{References}

[1] E. Wong, "Surgical Site Infections,” In: D. G. Mayhall, Ed., Hospital Epidemiology and Infection Control, 2nd Edition, Lippincott, Philadelphia, 1999, pp. 189-210.

[2] R. Plowman, N. Graves, M. A. S. Griffin, J. A. Roberts, A. V. Swan, B. Cookson, et al., "The Rate and Cost of Hospital-Acquired Infections Occurring in Patients Admitted to Selected Specialties of a District General Hospital in England and the National Burden Imposed," Journal of Hospital Infection, Vol. 47, No. 3, 2001, pp. 198-209. doi:10.1053/jhin.2000.0881

[3] L. J. Prokuski, "Selecting an Appropriate Prophylactic Antibiotic Agent; Reduce Surgical Site Infections with Appropriate Prophylactic Antibiotic Use,” American Academy of Orthpaedic Surgeons Bulletin, 2005.

[4] O. M. Lidwell, R. A. Elson, E. J. Lowbury, W. Whyte, R. Blowers, S. J. Stanley, et al., "Ultraclean Air and Antibiotics for Prevention of Postoperative Infection. A Multicenter Study of 8052 Joint Replacement Operations," Acta Orthopaedica Scandinavica, Vol. 58, No. 1, 1987, pp. 4-13. doi:10.3109/17453678709146334

[5] National Institute for Health and Clinical Excellence, "Surgical Site Infection: Prevention and Treatment of Surgical Site Infection,” October 2008.

[6] Scottish Intercollegiate Guidelines Network, "Antibiotic Prophylaxis in Surgery: A National Clinical Guideline,” Guideline No. 104, July 2008.

[7] T. Flinkkila, J. Ristiniemi, P. Hyvonen and M. Hamalainen, "Surgical Treatment of Unstable Fractures of the Distal Clavicle: A Comparative Study of Kirschner Wire and Clavicular Hook Plate Fixation,” Acta Orthopaedica Scandanica, Vol. 73, No.1, 2002, pp. 50-53. doi:10.1080/000164702317281404

[8] H. Habernek and L. Schmid, "Technique and Results of Modified Percutaneous Bore Wire Osteosynthesis of the Distal Radius,” Unfallchirurg, Vol. 95, No.7, 1992, pp. 339-343. 
[9] D. G. Armstrong, G. R. Pupp and L. B. Harkless, “Our Fixation with Fixation: Are Screws Clinically Superior to External Wires in Distal First Metatarsal Osteotomies?" Journal of Foot and Ankle Surgery, Vol. 36, No. 5, 1997, pp. 353-355. doi:10.1016/S1067-2516(97)80035-X

[10] L. Karapinar, H. Ozturk, T. Altay and B. Kose, "Closed Reduction and Percutaneous Pinning with Three Kirschner Wires in Children with Type III Displaced Supracondylar Fractures of the Humerus," Acta Orthopaedica et Traumatologica Turcical, Vol. 39, No. 1, 2005, pp. 23-29.

[11] P. Devkota, J. A. Khan, B. M. Acharya, N. M. Pradhan, L. P. Mainali, M. Singh, et al., "Outcome of Supracondylar Fractures of the Humerus in Children Treated by Closed Reduction and Percutaneous Pinning," Journal of Nepal Medical Association, Vol. 47, No. 170, 2008, pp. 66-70.

[12] J. Battle and K. D. Carmichael, "Incidence of Pin Track Infections in Children's Fractures Treated with Kirschner Wire Fixation," Journal of Pediatric Orthopaedics, Vol. 27, No. 2, 2007, pp. 154-157. doi:10.1097/bpo.0b013e3180317a22

[13] T. Azzopardi, S. Ehrendorfer, T. Coulton and M. Abela, "Unstable Extra-articular Fractures of the Distal Radius: A Prospective, Randomised Study of Immobilisation in a Cast Versus Supplementary Percutaneous Pinning,” Brit- ish Journal of Bone and Joint Surgery, Vol. 87, No. 6, 2005, pp. 837-840. doi:10.1302/0301-620X.87B6.15608

[14] A. Rafique, S. Ghani, M. Sadiq and I. A. Siddiqui, "Kirschner Wire Pin Tract Infection Rates between Percutaneous and Buried Wires in Treating Metacarpal and Phalangeal Fractures," Journal of College of Physicians and Surgeons Pakistan, Vol. 16, No. 8, 2006, pp. 518-520.

[15] G. Schmidmaier, M. Lucke, B. Wildemann, N. P. Haas and M. Raschke, "Prophylaxis and Treatment of Implant-Related Infections by Antibiotic-Coated Implants: A Review," Injury, Vol. 37, Supplement 2, 2006, pp. S105-S112. doi:10.1016/j.injury.2006.04.016

[16] D. G. Hargreaves, S. J. Drew and R. Eckersley, "Kirschner Wire Pin Tract Infection Rates: A Randomized Controlled Trial between Percutaneous and Buried Wires," British Journal of Hand Surgery, Vol. 29, No. 4, 2004, pp. 374-376. doi:10.1016/j.jhsb.2004.03.003

[17] A. Lethaby, J. Temple and J. Santy, "Pin Site Care for Preventing Infections Associated with External Bone Fixators and Pins," Cochrane Database of Systemic Review, No. 4, 2008, p. CD004551.

[18] M. M. Patterson, "Multicenter Pin Care Study," Orthopaedic Nursing, Vol. 24, No. 5, 2005, pp. 349-360. doi:10.1097/00006416-200509000-00011 